\title{
Safety and efficacy of canaloplasty versus trabeculectomy in treatment of glaucoma
}

\author{
Haifeng Liu ${ }^{1}$, Haitao Zhang ${ }^{2}$, Yanhua $\mathrm{Li}^{1}$ and Han Yu${ }^{1}$ \\ ${ }^{1}$ Department of Ophthalmology, The Third Affiliated Hospital of Xinxiang Medical University, Xinxiang, Henan, China \\ ${ }^{2}$ Department of Ophthalmology, The First Affiliated Hospital of Xinxiang Medical University, Xinxiang, Henan, China \\ Correspondence to: Haifeng Liv, email: liuhaifengdoc@126.com \\ Keywords: canaloplasty; trabeculectomy; glaucoma; meta-analysis \\ Received: November 17, $2016 \quad$ Accepted: January 11,2017 Published: January 19, 2017 \\ Copyright: Liu et al. This is an open-access article distributed under the terms of the Creative Commons Attribution License 3.0 (CC BY 3.0), \\ which permits unrestricted use, distribution, and reproduction in any medium, provided the original author and source are credited.
}

\section{ABSTRACT}

We assess the efficacy and safety of canaloplasty and trabeculectomy for treatment of glaucoma. We searched the China National Knowledge Infrastructure, PubMed, Web of Science, and WanFang databases for potentially eligible studies. Pooled risk ratio (RR) with $95 \%$ confidence interval (CI) was calculated using randomor fixed-effect models if appropriate. Eight studies were included for meta-analysis. There was no difference in intraocular pressure at 6 months (WMD $=0.97,95 \% \mathrm{CI}$ : -0.48-2.41). Intraocular pressure in canaloplasty group 12 months after operation was higher than in trabeculectomy group (WMD $=1.90,95 \% \mathrm{CI}: 0.12-3.69), P<$ $0.05)$. The canaloplasty group showed higher success rate than trabeculectomy group $(\mathrm{RR}=0.86,95 \% \mathrm{CI}: \mathbf{0 . 7 7 - 0 . 9 7 )}$. The canaloplasty group was more likely to have hyphema $(R R=2.96,95 \% C I: 1.51-5.83), P<0.05)$ than trabeculectomy group $(R R=$ $0.24,95, \mathrm{CI}(0.06-0.89), P<0.05]$. The incidence of and hypotony and postoperative choroid abnormalities in canaloplasty group was significantly lower than that in trabeculectomy group ( $R R=0.30,95 \% C I: 0.11-0.83$; $R R=0.24,95 \% C I: 0.09-0.66), P$ $<0.05$ ). Both trabeculectomy and canaloplasty can significantly reduce the intraocular pressure in glaucoma patients at 12 months after operation, trabeculectomy leads a more marked IOP decrease than canaloplasty at the cost of a higher complication rate and more demanding for postoperative care.

\section{INTRODUCTION}

Trabeculectomy has long been considered to be the gold standard procedure of lowering intraocular pressure in patients with glaucoma $[1,2]$. It involves draining aqueous humor from the anterior chamber into the subconjunctival spaces through a sclerostomy and requires full-thickness penetration of the anterior chamber under a partial-thickness scleral flap [3]. However, trabeculectomy can still be accompanied by some complications such as hyphemia [4-7], hypotony [8, 9], choroidal detachment [10-12], bleb leaks [13-15], and hemorrhaging. These complications are usually increased by using antifibrotics such as 5-fluorouracil, but the rate of short-term succus is relatively low without them [16]. Great efforts have been made for improving the safety and efficiency of glaucoma surgery. Some non-penetrating procedures such as canaloplasty have been reported. In this procedure, intraocular pressure reduction is achieved by opening previously non-functional areas of the outflow system: a 10-0 prolene suture is placed and pulled within Schlemm's canal, thus facilitating outflow through collector channels and aqueous veins $[17,18]$.

Recently, some researchers conducted some controlled trials to compare the safety and efficacy of canaloplasty versus trabeculectomy in treatment of glaucoma. However, the results still remain to be inconsistent. To our knowledge, the quality and consistency of epidemiological evidence on the topic have not been systematically investigated, which is an important gap in our understanding of safety and efficacy of canaloplasty versus trabeculectomy in treatment of glaucoma. With recently accumulated evidences, therefore, we performed a meta-analysis to compare the 
safety and efficacy of canaloplasty versus trabeculectomy in treatment of glaucoma. We hope this results could provide some support for clinical practice.

\section{RESULTS}

\section{Search results and study characteristics}

110 records were left after duplicates removed identified through the initial literature search. After review of the titles and abstracts, we excluded 84 records because they were not related topics. After review of the remaining 28 articles in full, 8 articles met all of the inclusion criteria and entered the final qualitative and quantitative synthesis [19-26]. The selection flow was shown in Figure 1.

The main characteristics of the included studies were presented in Table 1. These studies were published between 2010 to 2015 . The sample size was from 30 to 79. 3 studies were from German, 3 ones were from USA,
1 in Italy, and 1 in China. Of 8 studies, 4 are retrospective study and 4 are prospective studies. Only one study was conducted among infant [26], and others were among adults.

\section{Assessment of quality}

The NOS of all included studies ranged from 6 to 8 points. The average NOS score of the studies was 7. An additional file shows this in more detail (Supplementary Table S1).

\section{Pooled results}

\section{Intraocular pressure}

Figure 2 presents the pooled intraocular pressure results. This process includes 129 patients with canaloplasty and 179 patients with trabeculectomy. The random-effects models were used at 6 and 12 months

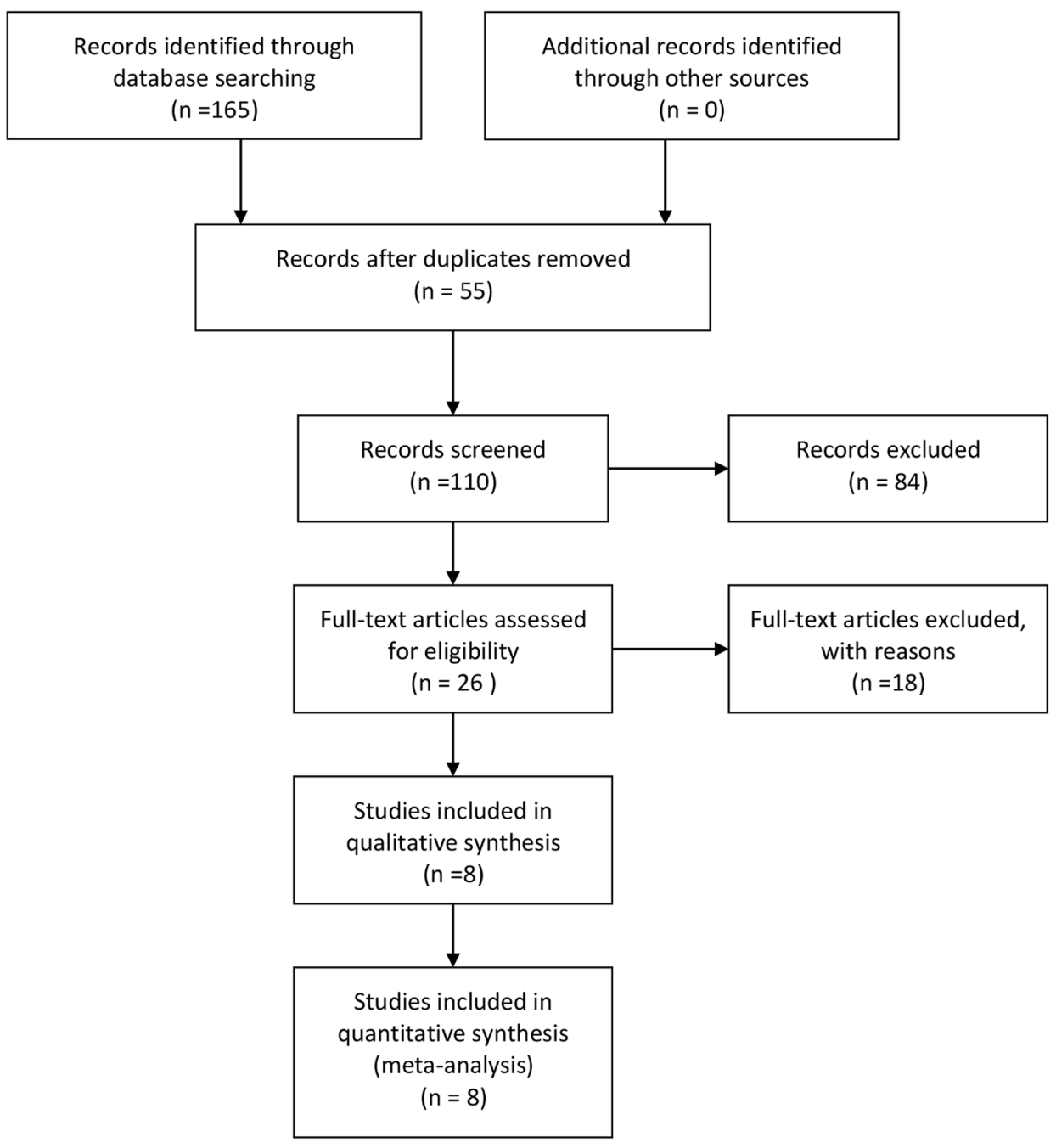

Figure 1: The process of study selection 
Table 1: General characteristics of studies included in the meta-analysis

\begin{tabular}{ccclcccc}
\hline Author & Year & Country & Study design & $\begin{array}{c}\text { Age } \\
\text { (CP/TE) }\end{array}$ & $\begin{array}{c}\text { Follow-up } \\
\text { (month) }\end{array}$ & $\begin{array}{c}\text { Sample size } \\
\text { (CP/TE) }\end{array}$ & NOS \\
\hline Schoenberg & 2015 & USA & Prospective cohort & $65.7 / 67.1$ & 12 & $36 / 41$ & 7 \\
Quaranta & 2014 & Italy & Prospective cohort & $63.8 / 63.0$ & 12 & $26 / 26$ & 6 \\
Thederan & 2014 & German & Retrospective cohort & $65.6 / 68.6$ & 12 & $18 / 22$ & 7 \\
Bruggemann & 2013 & German & Retrospective cohort & $58.7 / 59.5$ & 12 & $15 / 15$ & 7 \\
Matlach & 2013 & German & Prospective cohort & $63.2 / 60.1$ & 12 & $19 / 20$ & 8 \\
Bruggemann & 2012 & USA & Retrospective cohort & $-/-$ & 12 & $21 / 48$ & 6 \\
Ayyala & 2011 & USA & Prospective cohort & $65.6 / 68.6$ & 12 & $33 / 46$ & 8 \\
Huang & 2010 & China & Prospective cohort & $25.2 / 23.5$ & 12 & $23 / 28$ & 7 \\
\hline
\end{tabular}

after operation because the heterogeneity were high $\left(I^{2}=\right.$ $66 \%$ and $I^{2}=84 \%$ ). At 6 months, intraocular pressure of canaloplasty group was not prior to that in trabeculectomy group $(\mathrm{WMD}=0.97,95 \% \mathrm{CI}$ : $-0.48-2.41$, Figure 1$)$. The differences showed no significant. However, intraocular pressure of canaloplasty group were higher than in trabeculectomy group $(\mathrm{WMD}=1.90,95 \% \mathrm{CI}: 0.12-3.69$, Figure 2).

\section{Success rate}

Six studies reported the succus rate of intraocular pressure control, including 148 canaloplasty patients and 173 trabeculectomy patients. The results from fixed- effect models showed that success rate of canaloplasty group was lower than that of trabeculectomy group (RR $=0.86,95 \% \mathrm{CI}: 0.77-0.97, P=0.010$, Figure 3$)$, with low heterogeneity $\left(I^{2}=18 \%\right)$.

\section{Complications}

Six articles reported that adverse reactions and complications during follow-up, including hyphema, hypotony, and choroidal detachment. Five studies reported that incidence rate of hyphema. The heterogeneity within studies is low $\left(I^{2}=56 \%, I^{2}=0.0 \%, I^{2}=0.0 \%\right)$, and the fixed-effects models were conducted for these pooled results. Our results show canaloplasty group has a high

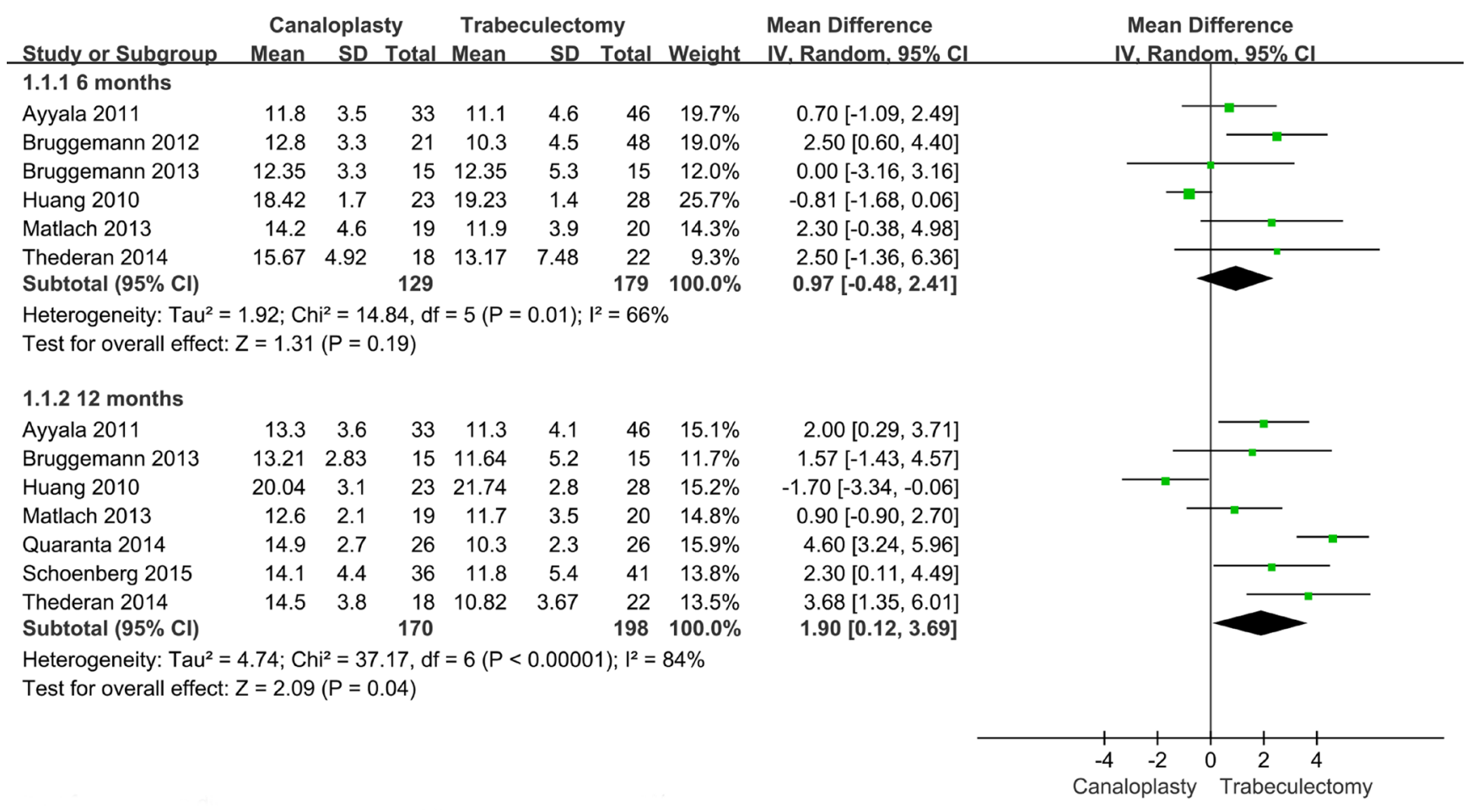

Figure 2: Forest plot of intraocular pressure at 6 and 12 months after operations 
risk of hyphema compared with trabeculectomy group (RR $=2.96,95 \% \mathrm{CI}: 1.51-5.83)$. However, canaloplasty group has lower risk of hypotony and choroidal detachment (RR $=0.30,95 \% \mathrm{CI}: 0.11-0.83 ; \mathrm{RR}=0.24,95 \% \mathrm{CI}: 0.09-0.66)$ compared with trabeculectomy group. The Figure 4 gives the specific results.

\section{Sensitivity analysis and publication bias}

We conducted sensitivity analysis through excluding some certain studies. The results did not alter exactly (Supplementary Figure 1). We use the Egger's and Begger'

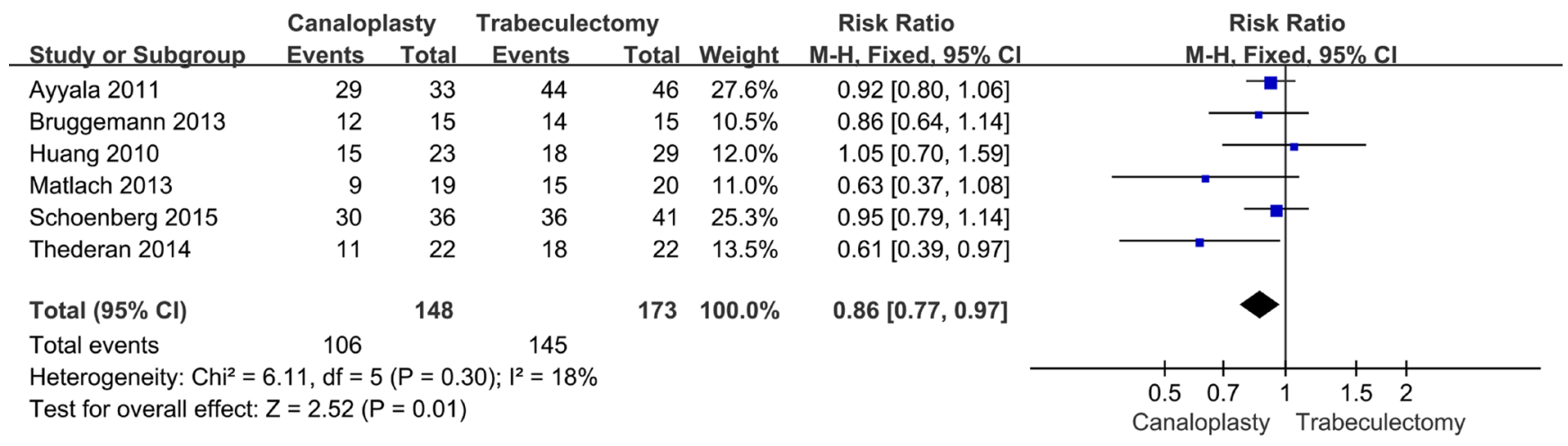

Figure 3: Succus rate of intraocular pressure control between two group.

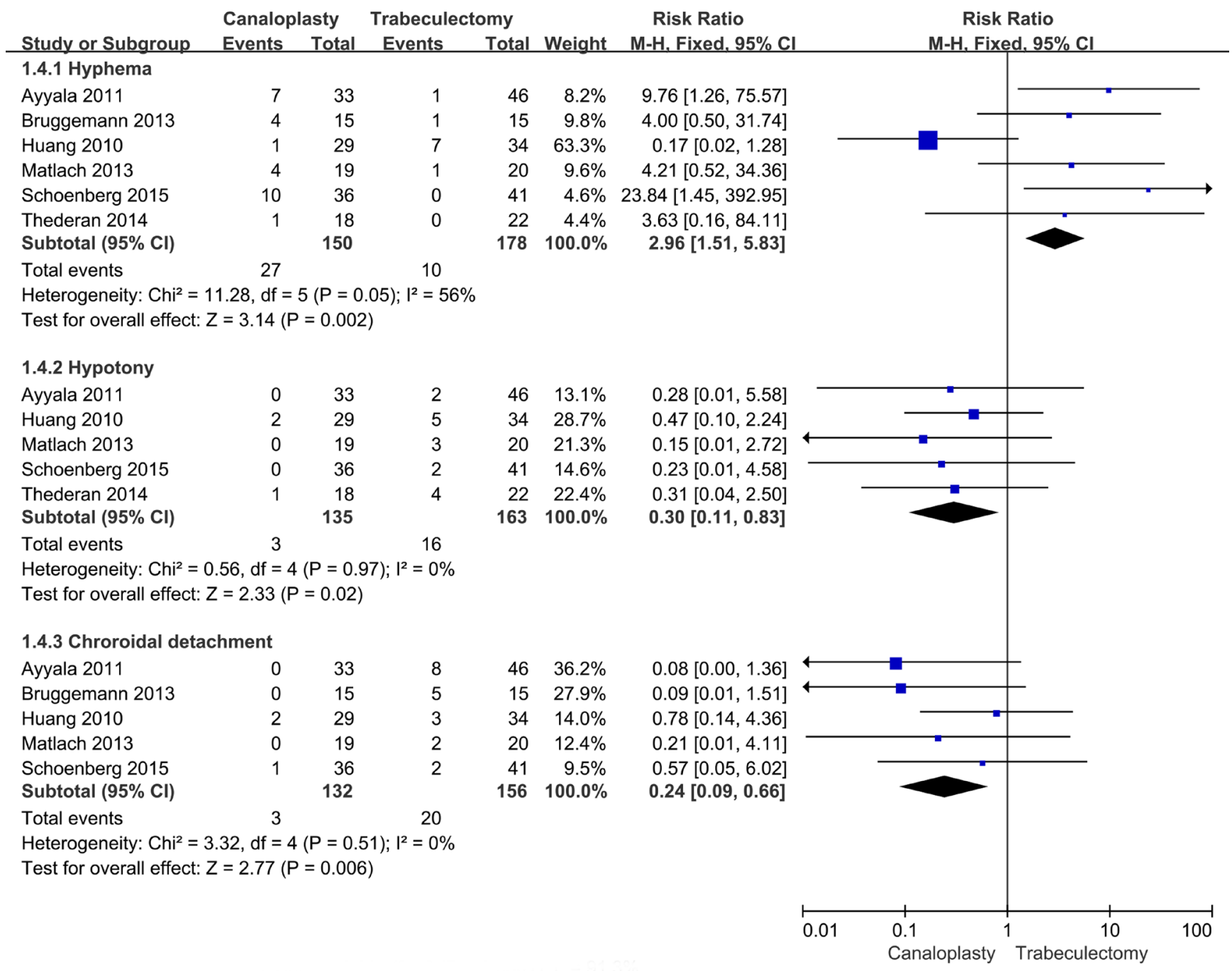

Figure 4: Forest plot of complications risk between two group. 
s to examine the publication. Egger's test suggested that publication bias did not exist $(\mathrm{t}=0.560, P=0.600)$. Also, the Begger's test give a similar result $(\mathrm{Z}=0.300, P=$ 0.764). The results are presented in Figure 5.

\section{DISCUSSION}

The present study found that trabeculectomy could reduce the intraocular pressure more obviously than canaloplasty at 12 months after operation. Trabeculectomy group have higher succus rate of intraocular pressure control while canaloplasty group are more likely to have hyphema compared with trabeculectomy group, but less possible to have hypotony and choroidal detachment.

Reducing intraocular pressure for glaucoma patients is the primary concern of operation therapy, with the aim of alleviating the optic nerve pressure. Reducing intraocular pressure is quite important for glaucoma treatment. Heijl reported that glaucoma progression had a 19 percent reduced risk when intraocular pressure decrease by $1 \mathrm{~mm}$ $\mathrm{Hg}$. Trabeculectomy operation is the standard treatment methods, but many complications would appear after operation and require more postoperative intervention. In canaloplasty procedure, the increased physiological drainage of trabecular meshwork archived by opening or expanding Schlemm's canal, without functional filtering bleb [27]. This procedure can avoid postoperative infection of filtering bleb and nursing, and theoretically can reduce the occurrence of postoperative complications and intervention therapy. If canaloplasty method can be proved to have similar of reducing intraocular pressure like trabeculectomy, and have less complications, these will have great important clinical significance. Currently, some clinical controlled trials are conducted to evaluate the safety and efficacy of comparing the canaloplasty versus trabeculectomy. However, the results remain inconsistent. Schoenberg reported that canaloplasty and trabeculectomy both achieved significant reduction in intraocular pressure and improvement in VA at 12 months with comparable success rates. trabeculectomy achieved a statistically greater median percentage decrease in intraocular pressure, but the 2 procedures resulted in comparable mean IOP at 12 months [19]. Thederan reported that the mean intraocular pressure in the trabeculectomy and canaloplasty groups reduced from 23.91 to $10.82 \mathrm{mmHg}$ and from $23.68 \mathrm{mmHg}$ to $14.50 \mathrm{mmHg}$, respectively. The postoperative complications and interventions between the two groups did not reveal a statistically significant difference. Complete success was achieved in 18 eyes $(81.8 \%)$ after trabeculectomy and in 11 eyes $(50.0 \%)$ after canaloplasty [21]. Ayyala, found that the mean percentage reduction in intraocular pressure from preoperative values at 12 months after surgery was $32 \%$ for the canaloplasty group compared with $43 \%$ for the trabeculectomy group. The median reduction in the number of medications at 12 months' follow-up was 3 in the trabeculectomy group and 2 in the canaloplasty group. But the differences were not significant. A higher percentage of patients treated with canaloplasty than trabeculectomy (36\% vs. $20 \%)$ required postoperative medications, although this also did not attain significance $(P=0.12)$. Failure rate based on intraocular pressure was $12.1 \%$ for the canaloplasty group and $4.3 \%$ for the trabeculectomy group. There was still no difference in surgical failure rates between the canaloplasty and trabeculectomy [25].

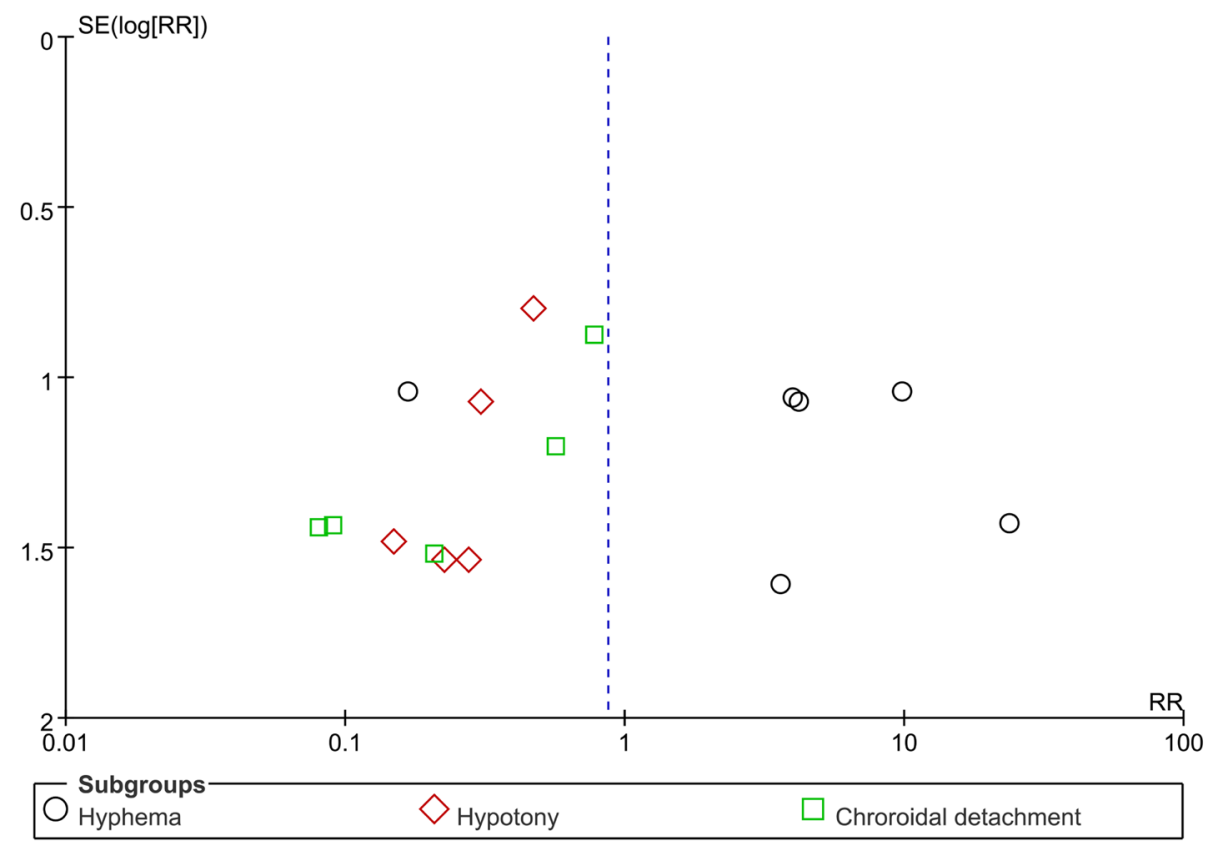

Figure 5: Funnel plot for publication bias assessment. 
The present study gives more powerful results Our findings provide support for clinical practice. Based on the present evidences, we propose that trabeculectomy operation should be applied for glaucoma patients with relatively high normal intraocular pressure. This method could improve the succus rate. Our results found that patients who received trabeculectomy had a higher risk of hypotony and choroidal detachment, indicating that canaloplasty operation could be used for patients who tended to have such complications like this. Although canaloplasty group have a higher risk of hyphema compared with trabeculectomy group, this complication usually did not carry out intervention because hyphema could be absorbed 1-3 weeks after operation [28].

Strengths of this meta-analysis included its validated and systematic review methods following PRISM guidelines and its high quality of included studies, with an average score of 7 allowing for the impossibility of one single study. These strengths make it possible for the present study to minimize the potential bias as much as possible, which is of great importance in the study design of observational studies. However, there are still several limitations needed to be addressed. First, the study number in this meta-analysis is limited, it is possible to have some bias. Second, six of included studies are from Caucasian, and only 2 of them are from Asian. The present results are limited in applying other population setting. Third, the assessment of effective are between 6 and 12 months after operation. More long-term follow-up are required to compare the long-term therapeutic effect. Finally, we can't assess the impact of the other clinically meaningful end points, such as automated perimetry, frequency doubling technology perimetry, scanning laser polarimetry, and confocal scanning laser ophthalmoscopy, because of sparse and inconsistent reporting across studies.

In conclusion, this meta-analysis of eight studies suggests that trabeculectomy method can significantly reduce the intraocular pressure better than canaloplasty method in glaucoma patients after operation, trabeculectomy leads a more marked IOP decrease than canaloplasty at the cost of a higher complication rate and more demanding for postoperative care.

\section{MATERIALS AND METHODS}

We performed this study in accordance with the Preferred Reporting Items for Systematic Reviews and Meta-Analyses (PRISMA) statement. No ethical statements are required for this study (Table S2).

\section{Literature search}

We searched China National Knowledge Infrastructure (CNKI), PubMed, Web of Science, and WanFang databases from inception to December 2016 for eligible studies, using the following Medical Subject Heading (MeSH) terms and keywords: canaloplasty, trabeculectomy, and glaucoma. Language was restricted in English and Chinese. We also checked the reference lists of previous related reviews and included studies for potentially eligible studies.

\section{Selection criteria}

We included the published studies that must meet the following criteria: (1) Type of study design: Clinical study with controls (No matter whether to use blind method or lost follow-up). (2) Study subject: patients with glaucoma. (3) Intervention: study with canaloplasty and trabeculectomy operation. (4) Outcomes: study with rate of efficient, intraocular pressure at 6 and 12 months after operation, higher success rate, and appearance of complications (hyphema, hypotony, and choroidal detachment).

The following studies are excluded: (1) patients with previous glaucoma operation or other intraocular surgery. (2) study cannot provide sufficient data for extraction and analyses or can't obtain original data from authors. (3) replicates articles are excluded.

Two investigators independently conducted the initial search, deleted duplicate records, screened the titles and abstracts for relevance and identified records as included, excluded or requiring further assessment.

\section{Data extraction and assessment of quality}

The following information was extracted from each study: the first author, publication year, country, study design, age (control and trial), period of follow-up, and sample size (two groups), intraocular pressure at 6 and 12 months after operation, success rate of operation, and some complications (hyphema, hypotony, and choroidal detachment) shown in results part.

The studies included in the meta-analysis were prospective or retrospective studies. We used NewcastleOttawa Scales (NOS) to assess the study quality [29]. The NOS includes 3 categories (selection, comparability and outcome) with 9 items. We evaluated the quality of studies as the following criteria: priority (7-8 adequate items), high quality (equal or more than 5 items), low quality (less than 5 items) and extreme low quality (no description of study methods).

\section{Statistical analysis}

We use the Review Manager software (RevMan version 5.2; Nordic Cochrane Centre, Cochrane Collaboration) and Stata 12.0 (Stata Corporation, College Station, TX, USA) to complete all analyses. We assessed 
the heterogeneity within the studies using the $I^{2}$ statistic and Cochran Q Chi-square test [30]. We considered heterogeneity to be substantial if the $I^{2}$ value was greater than $50 \%$. We pooled the data using the random-effects models or fixed-effects models. For continuous variables, we calculated standardized mean differences (WMDs) with 95\% CIs. For dichotomous data, we calculated relative risks (RRs) with 95\% confidence intervals (CIs). Sensitivity analyses and publication bias were evaluated using the Stata 12.0. A $p$ value of less than 0.05 was considered to be statistically significant.

\section{Abbreviations}

CNKI, China National Knowledge Infrastructure; MsSH, Medical Subject Heading; NOS, NewcastleOttawa Scales; WMD, weighted mean difference; RR, relative risk; CI, confidence interval; PRISMA, Preferred Reporting Items for Systematic Reviews and MetaAnalyses

\section{ACKNOWLEDGMENTS}

LHF designed this study and contributed substantially to the design of the search strategy. LHF and ZHT searched and selected the trials and extracted data. LHF, LYH and YH performed the analysis and interpreted the data. LHF wrote the manuscript. LHF and YH critically reviewed the manuscript. ZHT and LYH participated in the data extraction and critically revised it. LHF and ZHT proofread the final version. All authors read and approved the final manuscript.

\section{CONFLICTS OF INTEREST}

The authors declare that they have no conflicts of interest.

\section{REFERENCES}

1. Netland PA. Nonpenetrating glaucoma surgery. Ophthalmology. 2001; 108:416-421.

2. Cairns JE. Trabeculectomy. Preliminary report of a new method. AM J Ophthalmol. 1968; 66:673-679.

3. Razeghinejad MR, Fudemberg SJ and Spaeth GL. The changing conceptual basis of trabeculectomy: a review of past and current surgical techniques. Surv Ophthalmol. 2012; 57:1-25.

4. Kaplowitz K, Nobe M, Abazari A and Honkanen R. Trabeculectomy for traumatic hyphema in sickle cell trait. Semin Ophthalmol. 2015; 30:297-304.

5. Nakatake S, Yoshida S, Nakao S, Arita R, Yasuda M, Kita T, Enaida H, Ohshima Y and Ishibashi T. Hyphema is a risk factor for failure of trabeculectomy in neovascular glaucoma: a retrospective analysis. BMC Ophthalmol. 2014; 14:55.

6. Kojima S, Inatani M, Shobayashi K, Haga A, Inoue T and Tanihara H. Risk factors for hyphema after trabeculectomy with mitomycin C. J Glaucoma. 2014; 23:307-311.

7. Mannino G, Verrilli S, Calafiore S, Ciarnella A, Cutini A, Mannino C, Perdicchi A and Recupero SM. Evaluation of recurrent hyphema after trabeculectomy with ultrabiomicroscopy 50-80 MHz: a case report. BMC Res Notes. 2012; 5:549.

8. Sihota R, Angmo D, Sen S, Gupta V, Dada T and Pandey RM. The Long-term Outcome of Primary "Bleb-sparing, Epithelial Exchange" in Dysfunctional Filtering Blebs. J Glaucoma. 2016; 25:571-578.

9. Jongsareejit B, Tomidokoro A, Mimura T, Tomita G, Shirato $\mathrm{S}$ and Araie M. Efficacy and complications after trabeculectomy with mitomycin $\mathrm{C}$ in normal-tension glaucoma. Jap J Ophthalmol. 2005; 49:223-227.

10. Liu S, Sun LL, Kavanaugh AS, Langford MP and Liang C. Recurrent annular peripheral choroidal detachment after trabeculectomy. Case Rep Ophthalmol. 2013; 4:192-198.

11. Haga A, Inatani M, Shobayashi K, Kojima S, Inoue $T$ and Tanihara H. Risk factors for choroidal detachment after trabeculectomy with mitomycin C. Clin Ophthalmol. 2013; 7:1417-1421.

12. Silva NA and Silva FA. [Late and recurrent serous choroidal detachment after trabeculectomy: case report]. [Article in Portuguese]. Arq Bras Oftalmol. 2009; 72:552-555.

13. Fluorouracil Filtering Surgery Study one-year follow-up. The Fluorouracil Filtering Surgery Study Group. Am J Ophthalmol. 1989; 108:625-635.

14. Lin AP, Chung JE, Zhang KS, Chang MM, Orengo-Nania $\mathrm{S}$, Gross RL and Chang PT. Outcomes of surgical bleb revision for late-onset bleb leaks after trabeculectomy. J Glaucoma. 2013; 22:21-25.

15. Sagara H, Iida T, Saito K, Noji H, Ogasawara M and Oyamada H. Conservative treatment for late-onset bleb leaks after trabeculectomy with mitomycin $\mathrm{C}$ in patients with ocular surface disease. Clin Ophthalmol. 2012; 6:12731279.

16. Wilkins $\mathrm{M}$, Indar $\mathrm{A}$ and Wormald $\mathrm{R}$. Intra-operative mitomycin $\mathrm{C}$ for glaucoma surgery. Cochrane Database Syst Rev. 2005; 4: D2897.

17. Cameron B, Field M, Ball S and Kearney J. Circumferential viscodilation of Schlemms canal with a flexible microcannula during non-penetrating glaucoma surgery. Digit J Ophthalmology. 2006; 12:1-9.

18. Shingleton B, Tetz M and Korber N. Circumferential viscodilation and tensioning of Schlemm canal (canaloplasty) with temporal clear corneal phacoemulsification cataract surgery for open-angle glaucoma and visually significant cataract: one-year results. J Cataract Refract Surg. 2008; 34:433-440.

19. Schoenberg ED, Chaudhry AL, Chod R, Zurakowski D and 
Ayyala RS. Comparison of Surgical Outcomes Between Phacocanaloplasty and Phacotrabeculectomy at 12 Months' Follow-up: A Longitudinal Cohort Study. J Glaucoma. 2015; 24:543-549.

20. Quaranta L, Biagioli E, Riva I, Tosoni C, Brusini P, Centofanti M, Katsanos A, Floriani I and Konstas AG. Effect of trabeculectomy and canaloplasty on intra-ocular pressure modifications after postural changes in open-angle glaucoma. Acta Ophthalmol. 2014; 92: e498-e499.

21. Thederan L, Grehn F and Klink T. [Comparison of canaloplasty with trabeculectomy]. [Article in German]. Klin Monbl Augenheilkd. 2014; 231:256-261.

22. Bruggemann A, Despouy JT, Wegent A and Muller M. Intraindividual comparison of Canaloplasty versus trabeculectomy with mitomycin $\mathrm{C}$ in a single-surgeon series. J Glaucoma. 2013; 22:577-583.

23. Matlach J, Freiberg FJ, Leippi S, Grehn F and Klink T. Comparison of phacotrabeculectomy versus phacocanaloplasty in the treatment of patients with concomitant cataract and glaucoma. Bmc Ophthalmol. 2013; $13: 1$.

24. Bruggemann A and Muller M. [Trabeculectomy versus canaloplasty-utility and cost-effectiveness analysis] [Article in German]. Klin Monbl Augenheilkd. 2012; 229:1118-1123.
25. Ayyala RS, Chaudhry AL, Okogbaa CB and Zurakowski D. Comparison of surgical outcomes between canaloplasty and trabeculectomy at 12 months' follow-up. Ophthalmology. 2011; 118:2427-2433.

26. Huang LF and Chi W. Efficacy and safety of canaloplasty versus trabeculectomy in treatment of infantilism glaucoma. Journal of Chinese Physician. 2010; 12:785-787.

27. Lewis RA, von Wolff K, Tetz M, Koerber N, Kearney JR, Shingleton BJ and Samuelson TW. Canaloplasty: circumferential viscodilation and tensioning of Schlemm canal using a flexible microcatheter for the treatment of open-angle glaucoma in adults: two-year interim clinical study results. J Cataract Refract Surg. 2009; 35:814-824.

28. Grieshaber MC, Pienaar A, Olivier J and Stegmann R. Canaloplasty for primary open-angle glaucoma: long-term outcome. Br J Ophthalmol. 2010; 94:1478-1482.

29. Wells GA, Shea B, O'Connell D, Peterson J, Welch V, Losos M, Tugwell P. The Newcastle-Ottawa Scale (NOS) for assessing the quality if nonrandomized studies in meta-analyses. http://www.ohri.ca/programs/clinical_ epidemiology/oxford.asp. Accessed in December 21, $201 \overline{6}$

30. Higgins JP, Thompson SG, Deeks JJ, Altman DG. Measuring inconsistency in meta-analyses. BMJ. 2003; 327:557-560. 